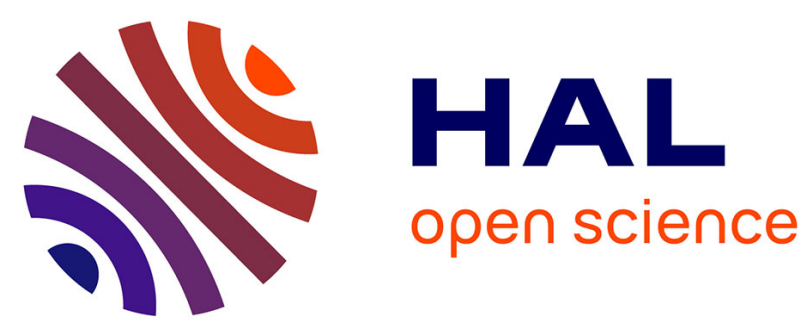

\title{
Goal-Driven Approach to Model Interaction between Viewpoints of a Multi-View KDD process
}

El Moukthtar Zemmouri, Hicham Behja, Brahim Ouhbi, Brigitte Trousse, Abdelaziz Marzak, Youssef Benghabrit

\section{- To cite this version:}

El Moukthtar Zemmouri, Hicham Behja, Brahim Ouhbi, Brigitte Trousse, Abdelaziz Marzak, et al.. Goal-Driven Approach to Model Interaction between Viewpoints of a Multi-View KDD process. 4th Conference on next generation networks \& Services, Dec 2012, Faro, Portugal. hal-00769796

\section{HAL Id: hal-00769796 \\ https://hal.inria.fr/hal-00769796}

Submitted on 3 Jan 2013

HAL is a multi-disciplinary open access archive for the deposit and dissemination of scientific research documents, whether they are published or not. The documents may come from teaching and research institutions in France or abroad, or from public or private research centers.
L'archive ouverte pluridisciplinaire HAL, est destinée au dépôt et à la diffusion de documents scientifiques de niveau recherche, publiés ou non, émanant des établissements d'enseignement et de recherche français ou étrangers, des laboratoires publics ou privés. 


\title{
Goal Driven Approach to Model Interaction between Viewpoints of a Multi-view KDD Process
}

\author{
EL Moukhtar Zemmouri ${ }^{(1)}$, Hicham Behja ${ }^{(1,2)}$, Brahim Ouhbi ${ }^{(1)}$, Brigitte Trousse ${ }^{(2)}$, \\ Abdelaziz Marzak $^{(3)}$, Youssef Benghabrit ${ }^{(1)}$ \\ (1) Ecole Nationale Supérieure d'Arts et Métiers - Meknes, Morocco \\ ezemouri@yahoo.fr, h_behja@yahoo.com, \\ ouhbib@yahoo.co.uk, you_benghabrit@yahoo.fr \\ ${ }^{(2)}$ Equipe-projet AxIS, INRIA Sophia Antipolis - Méditerranée, France \\ brigitte.trousse@inria.fr \\ ${ }^{(3)}$ Faculté des Sciences Ben Msik, Casablanca, Morocco \\ marzak@hotmail.com
}

\begin{abstract}
A data mining project is usually held by several actors (domain experts, data analysts, KDD experts ...), each with a different viewpoint. In this paper we propose to enhance coordination and knowledge sharing between actors of a multiview KDD analysis through a goal driven modeling of interactions between viewpoints. After a brief review of our approach of viewpoint in KDD, we will first develop a Goal Model that allows identification and representation of business objectives during the business understanding step of KDD process. Then, based on this goal model, we define a set of relations between viewpoints of a multi-view analysis; namely equivalence, inclusion, conflict and requirement.
\end{abstract}

Keywords-component; KDD process; Viewpoint; Goal analysis; Ontologies; SWRL

\section{INTRODUCTION}

In recent decades, enterprises' information systems become more and more flooded by all kind of data: structured (databases, data warehouse), semi-structured (XML, server log files), and unstructured data (raw text, multimedia data). This has created new challenges for companies and for the scientific community. Including, how to understand and analyze such a mass of data to extract knowledge. Hence, KDD (Knowledge Discovery in Databases [1]) have rapidly changed from a research area into an industry standard (i.e. CRISP-DM: CrossIndustry Standard Process for Data Mining [2]). In [3], Kurgan and Musilek draw up a comprehensive survey of knowledge discovery and data mining standard process models.

But, available data mining tools (commercial as well as free tools) support end-users only with graphical and/or manual construction of KDD execution plans. This is done without taking into account analyst viewpoint and coordination within organization (coordination between domain experts, data analysts, and KDD experts). But this is time consuming, cost sensitive, and requires good experience and prior knowledge of data mining domain to successfully conduct a KDD project.

Our objective in this paper is to model and formalize interactions between different viewpoints of a multi-view KDD process (a KDD process held by several experts who analyze the same data with different viewpoints). This is done by introducing a set of semantic relations between viewpoints.
Our formalization is based on a goal driven approach and will allow us to enhance coordination, knowledge sharing and mutual understanding between different actors of a multi-view analysis, and reusability in terms of viewpoint of successful data mining experiences within an organization.

In the reminder of this introductory section we briefly recall our approach of viewpoint in KDD to fix definitions and context of our work. This approach was initiated by Behja et al. [4] [5] as part of Behja's doctoral thesis in the context of France-Morocco cooperation (Software Engineering Network) 1 .

A multi-view KDD process is usually held by one or more users who consequently manipulate several types of knowledge and know-how. They will have different objectives and preferences, different competences, and different visions of analyzed data, KDD methods and functions. In brief, they have different viewpoints. In this context, the KDD process will be guided by the analyst viewpoint [4] and several types of knowledge and expertise are incorporated (analyzed domain knowledge and analyst domain knowledge).

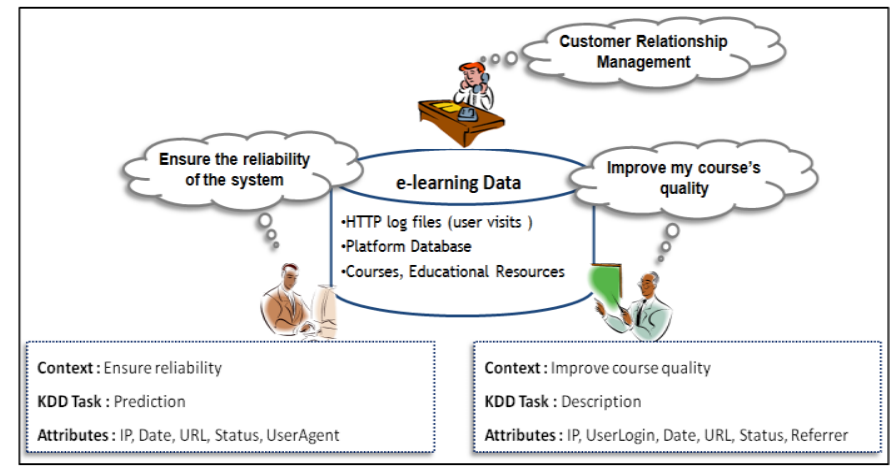

Figure 1. Multitude of viewpioints to analyse data from an e-learing system [6].

Figure 1 shows an example of a multi-view analysis of data from an e-learning system (that consists of HTTP log files, platform database, and courses material). These data can be analyzed by different actors of the system (teachers, learners,

\footnotetext{
${ }^{1}$ http://raweb.inria.fr/rapportsactivite/RA2003/axis/uid81.html
} 
administrator, marketing, KDD expert, data miner). The objective of a teacher (e.g. improve quality of a course) is not the same as the administrator's one (e.g. ensure system reliability). Attributes used for evaluating a course are different from those used for studying the reliability. Similarly, chosen data mining methods, techniques and tools will be different, and interpretation/deployment of data mining results depends on the analyst's viewpoint. Therefore, it is fundamental to take into account the viewpoint of each analyst and interaction between different viewpoints.

In previous works we have developed a Knowledge Model for multi-view KDD process [5] which is " $a$ specification of the information and knowledge structures and functions involved [during a multi-view analysis]" [7]. As shown in figure 2, our Knowledge Model integrating the viewpoint notion consists of four hierarchical sub-models structured in domain knowledge and strategic knowledge. Domain level describes the domain concepts and their relationships. The strategic level is based on the domain level and expresses how a task will be achieved.

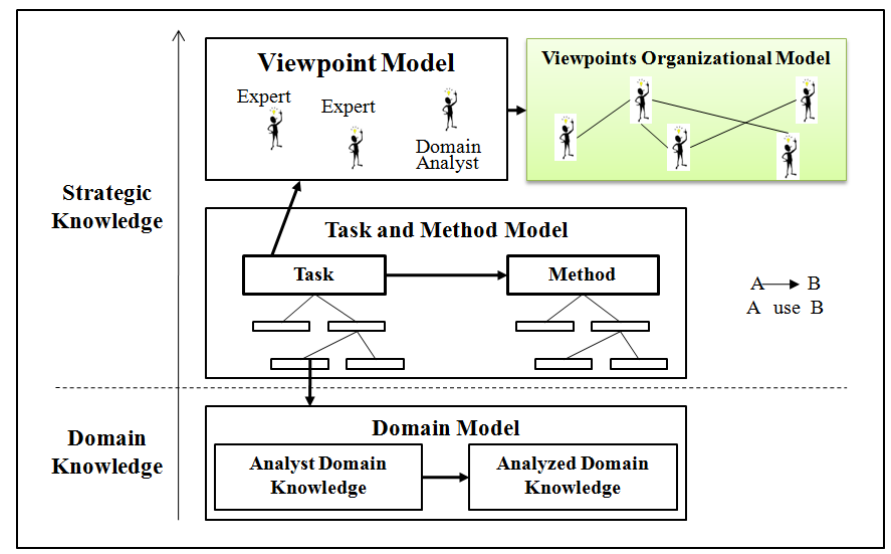

Figure 2. Knowledge Model for a multi-view KDD process [5].

The Domain Model concerns the studied domain concepts and the various relations between them. In our applicative context, that contains an e-learning ontology, platform database and HTTP log file structure. Task and Method Model describes the KDD process in terms of tasks and methods. We have formalized this model as a generic semi-formal ontology OntoECD [8]. Viewpoint Model is a conceptualization of a set of generic criteria that characterize a viewpoint in KDD [6]. These generic criteria were identified based on CRISP-DM reference model and formalized as a viewpoint ontology in OWL.

In this paper we will mainly focus on the development of the Viewpoint Organizational Model.

The remainder of the paper is organized as follows. In section 2 we will give a state of the art of works related to supporting users of KDD and managing knowledge in such process especially using ontologies. In section 3, we will develop a goal model that represents business objectives of different actors of a multi-view analysis, and formalize two types of relations between goals: AND/OR decomposition, and positive/negative influence. We will also illustrate our goal model within an example in e-learning. Based on this goal model, in section 4, we will define a set of semantic relations between viewpoints in KDD, especially: equivalence, conflict, and requirement. As stated before, the main purpose of these relations is to provide a means of coordination and mutual understanding between viewpoints actors. In section 6 , a case tool implementation to validate our approach is presented. Finally, we will draw some learned lessons in conclusion.

\section{RELATED WORK}

"An analyst is usually not a KDD expert but a person responsible for making sense of the data using available KDD techniques. Since the KDD process is by definition interactive and iterative, it is a challenge to provide a high-performance, rapid-response environment that also assists users in the proper selection and matching of appropriate tools and techniques to achieve their goals" [1]. This was a statement of Fayyad et al. in 1996 highlighting the challenge of supporting both expert and novice users of KDD due to its complexity. We notice also that during a KDD process (a data mining project according to CRISP-DM vocabulary) there will be several actors (domain expert, data analyst, data miner ...). The question we address in this paper is how to provide a framework allowing these actors to collaborate, share knowledge (about business domain and data mining techniques), and reuse each others' experiences.

Several works and standards have addressed the complexity of KDD with different approaches with the aim of supporting both expert and novice users. Most of these approaches are based on ontologies (either domain ontologies, or data mining ontologies). They offer the user the advantage to explore the large space of valid data mining processes [9][10], to discover and access distributed data mining services [11][10], to reuse successful data mining experiences [12], etc. But without taking into account the multi-view aspect of a KDD analysis.

One of the first ontologies proposed to support users of KDD is DAMON (Data Mining ONtology) [13] that is designed to simplify the development of distributed KDD applications on Grids. DAMON ontology concerns only the data mining phase of a KDD process, and offers a taxonomy for discovering tasks, methods and tools deemed more suitable for a given data mining goal.

In MiningMart project [12] a case-based reasoning (CBR) system to support end users during data preprocessing is proposed. This system is based on a meta-model (called M4) of KDD preprocessing chains that contains ontology for describing conceptual domain knowledge. In the same project, Euler [11] proposes a web-based platform (which is a case base containing MiningMart successful experiences) to publicly display preprocessing models in a structured way, together with descriptions about their business domains, goals, methods and results.

Bernstein et al., [9] propose an Intelligent Discovery Assistant (IDA) for valid data mining processes enumeration and ranking. IDA focuses mainly on preprocessing and data mining phases of the KDD process. It is based on a formal ontology that contains input/output, preconditions constraint, and performance (accuracy, complexity, and 
comprehensibility) of each data mining operator. This ontology allows selection and composition of data mining operators suitable for user's data and goal. A similar approach is proposed by Diamantini et al., [10] in a project called KDDVM (KDD Virtual Mart), which is a web services based system that aims to support users in the design of valid KDD process. It represents KDD operations as services which can be "annotated, introduced, accessed, described, composed and activated". KDDVM is based on KDDONTO ontology and concerns only data preprocessing and data mining steps.

A recent European project $\left(\mathrm{e}-\mathrm{LICO}^{2}\right.$ : e-Laboratory for Interdisciplinary Collaborative Research in Data Mining and Data-Intensive Science) deals with the problem of supporting users of KDD in a collaborative way [14]. One of the products of this project is eProPlan [15], an ontology based environment for planning KDD workflows. It is based on two ontologies DMWF-DMOP and uses IA planning techniques to automatically generate KDD execution plan for solving data mining problems. DMWF (Data Mining Work Flow Ontology) formalizes IO-objects, operators, goals, tasks and methods as well as the decomposition of tasks into methods and operators (this ontology is equivalent to our OntoECD ontology described in [8]). DMOP (Data Mining Optimization Ontology) provides a unified conceptual framework for analyzing data mining tasks, algorithms, models, datasets, workflows and performance metrics, and their relationships.

Our approach of supporting KDD users focuses on the reusability, coordination, and knowledge sharing between multi-users of a KDD process, rather than the automatic generation of KDD execution plan. In addition, we cover the whole phases of the KDD process (cf. 6 phases suggested by CRISP-DM reference model [2]).

In previous works we have addressed mainly three issues: integration of domain knowledge [6], annotation of KDD in terms of viewpoint [16], and viewpoint characterization and modeling [6].

In this paper we propose to focus on modeling interactions between viewpoints of multi-view KDD process. For this, our proposed model will be based on a goal driven approach [17].

\section{GOAL MODEL FOR MULTI-VIEW KDD PROCESS}

Goal driven analysis consists of the identification, modeling and analysis of goals and objectives that guide decisions and strategies of an organization at different levels. Goals are also defined to be a logical mechanism for identifying, organizing and justifying requirements [17].

Goal models have been used in Software Engineering [18] [19] and Data Warehousing [20] [21] [22] in order to represent users requirements, business objectives and design qualities. Indeed, eliciting requirements (as high level goals) early in the development process is crucial [19]. Requirements can be functional or non-functional. Non-functional requirements (also known as quality requirements) are defined as attributes or constraints of the system such as performance, security, and reliability [23].

2 e-LICO Project, http://www.e-lico.eu/
Goal models are recognized also to be useful for knowledge management systems which focus on strategic knowledge representation and reasoning [24][25]. In this case, goals are used to represent strategic objectives of an organization and to analyze and keep track of events and trends that may influence positively or negatively these objectives.

\section{A. Generic goal model for multi-view KDD process}

In the context of multi-view KDD process we propose to use the goal-driven approach to identify and represent business objectives (this is recognized to be the first task of the first step of CRISP-DM reference model) of a multi-view KDD process and to model interactions between viewpoints of stakeholders of these objectives. This will provide users with a methodological assistance during the business understanding step of KDD and make business objectives persistent for the other steps (especially evaluation and deployment of data mining results). In fact, as is the case for requirements traceability and persistence in data warehousing [21] and software development [26], we support here the necessity of storing early defined business objectives. Their presence can guide many decisions and solve many problems encountered at different stages of a KDD analysis. The persistence of goals, in our approach, is intended also to be a complement of metadata schema introduced by Behja et al. [16][5] to annotate and keep track of multi-view analysis.

Our proposed generic goal model for multi-view KDD process, represented as OWL ontology, is depicted in figure 3:

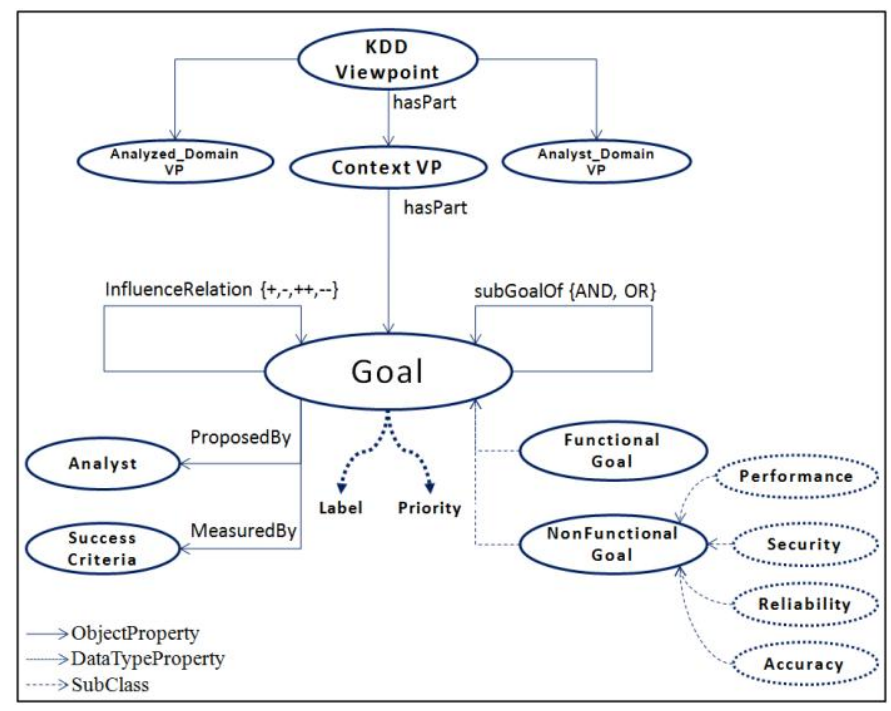

Figure 3. Generic Goal Model for multi-view KDD process.

During a data mining project, an analyst (actor of the system) may have one or more goals (these goals are considered as a part of the context associated with his/her viewpoint [6]). A goal can be either functional or nonfunctional. Functional goals reflect business objectives, while non-functional goals reflect constraints of the system (such as security, reliability, and performance) or technical constraints on the execution of KDD methods (such as accuracy, cost sensitivity, comprehensibility, runtime, number of techniques 
that form the execution plan, etc.). As an example: "Improve quality of a course" is a functional goal, "ensure reliability of the e-learning platform" is a non-functional one.

A goal may have two attributes (Data Type Property in OWL vocabulary) priority and label. Label indicates if a goal is satisfied or denied [25]. Priority indicates if a goal is mandatory or optional [21].

A goal is proposed by an actor (KDD expert, domain expert, and data miner are example of actors) and is measured by some success criteria.

In goal-driven approach, two types of reflexive relations (Object Property in OWL vocabulary) between goals are defined (especially for reasoning purposes) [25]: sub-goal relation and influence relation.

Sub-goal relation consists of the decomposition of a general goal into more specific sub-goals. For this relation two main sub-relations are used: AND and OR decomposition. Semantic of these relations is as follow:

- If a goal $G$ is AND-decomposed into sub-goals $G_{1}, G_{2}$, $\ldots, G_{n}$, then if all of the sub-goals are satisfied so is goal $G$.

- If a goal $G$ is OR-decomposed into sub-goals $G_{1}, G_{2}$, $\ldots, G_{n}$, then if at least one of the sub-goals is satisfied so is goal $G$.

To formalize these relations, in the case of binary decomposition, we define the following $\mathrm{SWRL}^{3}$ rules:

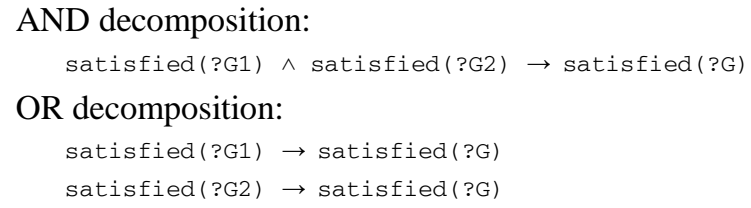

Notice that binary decomposition is not a restriction since it can be easily generalized into n-ary decomposition.

Influence relation: this type of relation models situations where a goal contributes positively or negatively towards the satisfaction/denial of another goal. It is described to be more qualitative relation than AND, OR-decomposition [25].

Influence relation has 4 sub-relations that are labeled $(+,-,++$, and --) with the following semantic:

- $\quad+\left(\mathrm{G}, \mathrm{G}^{\prime}\right)$ : if $G$ is satisfied then $G^{\prime}$ is partially satisfied.

- $\quad-\left(\mathrm{G}, \mathrm{G}^{\prime}\right)$ : if $G$ is satisfied then $G^{\prime}$ is partially denied.

- $\quad++\left(\mathrm{G}, \mathrm{G}^{\prime}\right)$ : if $G$ is satisfied then $G^{\prime}$ is fully satisfied.

- $\quad--\left(\mathrm{G}, \mathrm{G}^{\prime}\right)$ : if $G$ is satisfied then $G^{\prime}$ is fully denied.

To formalize these relations, we define the following SWRL rules:

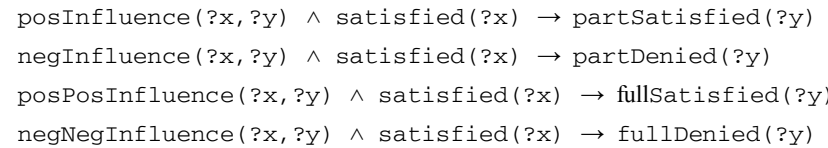

${ }^{3}$ SWRL: A Semantic Web Rule Language Combining OWL and RuleML, http://www.w3.org/Submission/SWRL/
Where (posInfluence, negInfluence, posPosInfluence, and negNegInfluence) represent respectively influence relations $(+$, ,-++ , and --) in OWL.

\section{B. An example}

In this subsection we will illustrate through an example in our applicative context e-learning. This example will be considered as an instance of the goal model presented in the previous sub-section.

Assume that we want to conduct a data mining project to analyze data from a web based e-learning platform. According to CRISP-DM methodology [27], the first and critical step of the project is business understanding, where business objectives must be identified, analyzed and then transformed into data mining tasks. In our case study, examples of such business objectives are: "improve course quality" form the teacher viewpoint, "manage users' relationship" from the marketing viewpoint, and "ensure reliability of the platform" from the admin viewpoint (see figure 1).

These business objectives are modeled using goals and goal relations (AND/OR decomposition, +/- influence) as illustrated in figure 4 below.

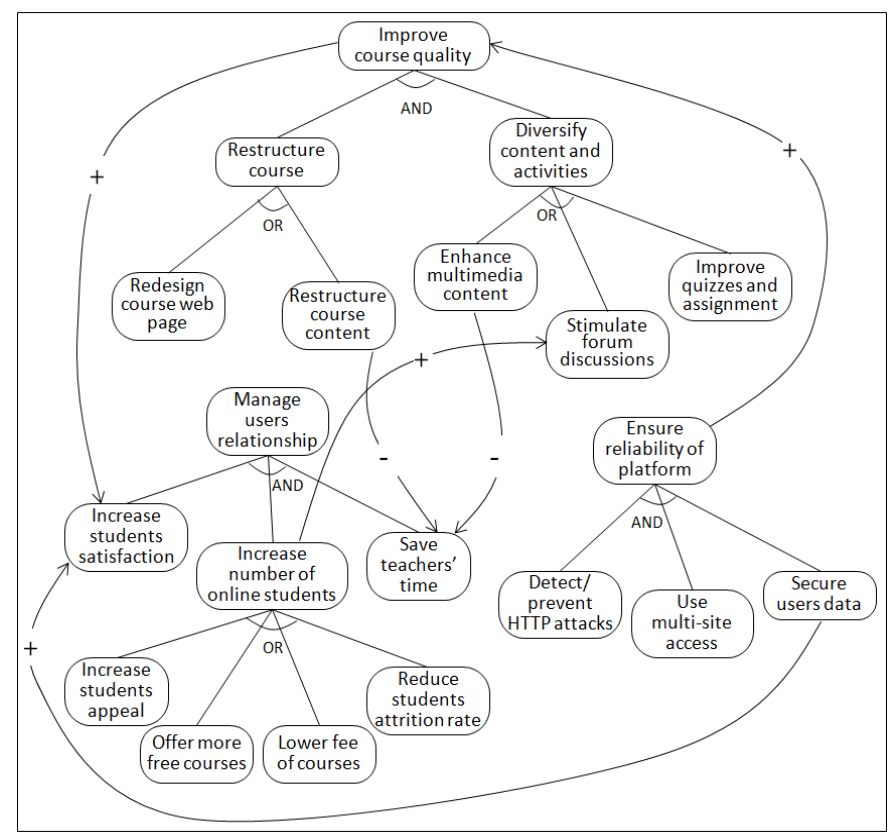

Figure 4. Example of a goal model for analysing a web based e-learining system.

The teacher goal "improve course quality" is ANDdecomposed to "restructure the course" and "diversify content and activities", and is positively influenced by the admin goal "ensure reliability of the platform" (i.e., satisfaction of the latter contribute positively towards the satisfaction of the former). The admin goal "ensure reliability of the platform" is AND-decomposed to "secure users data", "use multi-site access", and "detect and prevent HTTP attacks".

The decomposition and refinement of goals continue until we have sub-goals that are tangible [25] (i.e., they can be analyzed using a simple KDD task). 
One of the main issues in goal-driven analysis is goals identification. Even if there exist some attempts to guide the identification of goals (like eliciting goals from scenarios, eliciting goals by refinement, and eliciting goals by reuse [28]). We argue that, in the context of KDD, the assistance of domain experts is necessary to guide and validate the identification of goals and influences between them.

\section{VIEWPOINTS INTERACTION}

In most modeling paradigms for information systems (such as Entity Relationship modeling, Object Oriented modeling, and Conceptual Graphs), relations between objects are recognized to have a predominant role. In particular, relations express dependency between entities of a conceptual model. In addition, during a multi-view analysis, it is important to emphasize the interaction and interdependence between the various analyses according to different viewpoints. It is therefore necessary for our approach of knowledge representation in a multi-view KDD process to provide opportunities for expressing such interaction and dependency in terms of relations.

Based on the presented goal model and relationships between goals we introduce some relations between viewpoints of a multi-view analysis. The main purposes of these relations are to enhance coordination and mutual understanding between viewpoint stakeholders, and to allow reusability of KDD process in terms of viewpoint.

Let $\mathrm{VP}_{1}$ and $\mathrm{VP}_{2}$ be two different viewpoints during a multi-view analysis, $\mathrm{G}_{1}=\left\{\mathrm{G}_{11}, \mathrm{G}_{12}, \ldots, \mathrm{G}_{1 \mathrm{n}}\right\}$ goals associated with $V P_{1}, G_{2}=\left\{G_{21}, G_{22}, \ldots, G_{2 m}\right\}$ goals associated with $V P_{2}$, $\left\{\mathrm{g}_{11}, \mathrm{~g}_{12}, \ldots, \mathrm{g}_{1 \mathrm{i}}\right\}$ a subset of $\mathrm{G}_{1}$, and $\left\{\mathrm{g}_{21}, \mathrm{~g}_{22}, \ldots, \mathrm{g}_{2 \mathrm{j}}\right\}$ a subset of $\mathrm{G}_{2}$. We define and formalize equivalence, inclusion, conflict, and requirement relations between $\mathrm{VP}_{1}$ and $\mathrm{VP}_{2}$ as follow:

\section{- Equivalence:}

$V P_{1}$ is equivalent to $V P_{2}$ if:

Satisfaction of all of the goals associated with $\mathrm{VP}_{1}$ implies satisfaction of all of the goals associated with $\mathrm{VP}_{2}$ and vice versa.

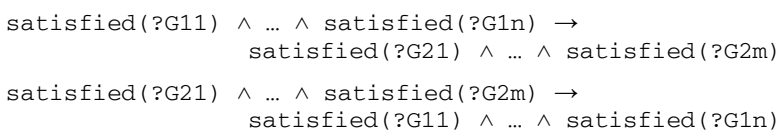

\section{- Inclusion:}

$V P_{1}$ includes $V P_{2}$ if:

Satisfaction of some of the goals associated with $\mathrm{VP}_{1}$ implies satisfaction of all of the goals associated with $\mathrm{VP}_{2}$.

satisfied(?g11) $\wedge \ldots \wedge$ satisfied(?g1i) $\rightarrow$

satisfied(?G21) $\wedge \ldots \wedge$ satisfied (?G2m)

\section{- Conflict:}

$V P_{1}$ is in conflict with $V P_{2}$ if

Satisfaction of some of the goals associated with $\mathrm{VP}_{1}$ implies denial of some of the goals associated with $\mathrm{VP}_{2}$.

satisfied(?g11) $\wedge \ldots \wedge$ satisfied(?g1i) $\rightarrow$ denied $(? g 21) \wedge \ldots \wedge$ denied $(? g 2 j)$

\section{- Requirement:}

$V P_{1}$ requires $V P_{2}$ if:

Satisfaction of some of the goals associated with $\mathrm{VP}_{1}$ requires satisfaction of all of the goals associated with $\mathrm{VP}_{2}$.

satisfied(?g11) $\wedge \ldots \wedge$ satisfied(?g1i) $\leftarrow$

satisfied (?G21) $\wedge \ldots \wedge$ satisfied (?G2m)

The equivalence relation serves mainly the purpose of reusability of KDD process in terms of viewpoint. In fact, it may be benefit within an organization to reuse successful data mining experiences to achieve different business objectives (goals) associated to different viewpoints (actors). This is done by comparing only the goal models associated with VP1 and VP2 without considering the technical details of the KDD execution plans.

The conflict, inclusion and requirement relations can be used to provide methodological assistance to a KDD user to achieve his/her goals. Especially tasks to be avoided in the case of conflict with another VP, or tasks to be partially reused in the case of inclusion and requirement.

\section{IMPLEMENTATION OVERVIEW}

To validate our approach, we are implementing a case tool in Java language that can be used by KDD users to design valid KDD process by reusing existing ones. As illustrated in figure 5, our assistant consists of 5 main components: a graphical user interface, a case based reasoner, a rule based reasoner, a KDD case base, and OWL ontologies.

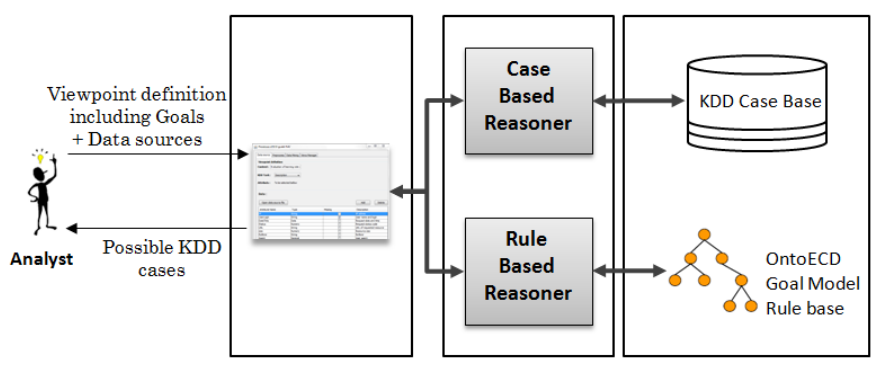

Figure 5. Architecture overview of our implementing tool.

OWL ontologies and rule base give a formal representation of knowledge involved during a KDD analysis, while the KDD case base holds detailed annotations [16] about successful data mining experiences (data sources characterization, goals instances, used data mining methods and their parameters, etc.). The case based reasoner provides a set of similar KDD cases based on the analyst definition of viewpoint (that includes goals identification and data sources) and using the previously defined relations between viewpoints. Reusability/adaptation of a KDD case is carried out by the inference capabilities of the rule based reasoner, which based on FaCT ++ reasoner [29] and SWRL.

\section{CONCLUSION}

To support users of multi-view KDD process within an organization, we have proposed a goal-based formal 
framework to model and formalize business objectives and interactions between viewpoints of a multi-view KDD process.

At first we have presented a goal model to identify and represent business objectives during the business understanding step of KDD. We defined also two reflexive relations within goals: AND/OR decomposition and influence relation. A precise semantic has been given for these relations as well as a formalization using SWRL language. This goal model has the benefit of assisting users during the early stage of the KDD process to clearly define business objectives of the data mining project, and then to make them persistent. This persistence will guide decisions at different steps of the KDD process, especially during the evaluation and deployment of data mining results.

Then, to formalize interaction and interdependence between various analyses according to different viewpoints, we have presented a set of semantic relations between viewpoints. We have defined equivalence, inclusion, conflict, and requirement relations. These relations allow us to enhance coordination, knowledge sharing and mutual understanding between different actors of a multi-view analysis, and reusability in terms of viewpoint of successful data mining experiences within an organization.

Finally we argue that our approach has the benefit of minimizing the cost of developing new KDD analyzes (since we can develop new analyzes from earlier), and providing a methodological assistance during KDD steps (especially about tasks and choices to be reused or avoided). The evaluation of our proposal is conducted by implementing a case tool and analyzing data and viewpoints from an e-learning system.

\section{REFERENCES}

[1] U. M. Fayyad, G. Piatetsky-Shapiro, and P. Smyth, "The KDD Process for Extracting Useful Knowledge from Volumes of Data," Communications of the ACM, vol. 39(11), p. 27-34, 1996.

[2] C. Shearer, "The CRISP-DM model: the new blueprint for data mining," Journal of Data Warehousing, Vol. 15(4), pp 13-19, 2000.

[3] L. A. Kurgan, and P. Musilek, "A survey of Knowledge Discovery and Data Mining process models," The Knowledge Engineering Review, Cambridge University Press, USA, Vol. 21(1), pp. 1-24, March 2006.

[4] H. Behja, B. Trousse, and A. Marzak, "Prise en compte des points de vue pour l'annotation d'un processus d'Extraction de Connaissances à partir de Données," In S. Pinson \& N. Vincent (Eds.), Revue des Nouvelles Technologies de l'Information (RNTI-E-3) : Vol. 1, pp. 245256, Cépaduès-Editions, 2005.

[5] H. Behja, Plateforme objet d'évaluation orientée point de vue d'un système d'information, Ph.D. thesis, Faculty of Science Ben M'sik, Casablanca, Morocco, February 2009.

[6] E. Zemmouri, H. Behja, A. Marzak, and B. Trousse, "Ontology-based Knowledge Model for Multi-view KDD Process," International Journal of Mobile Computing and Multimedia Communications (IJMCMC), Vol. 4(3), pp. 21-33, 2012.

[7] A. Schreiber, J. M. Akkermans, A. Anjewierden, R. Hoog, N. Shadbolt, W. Van de Velde, and B. J. Wielinga, Knowledge Engineering and Management: The CommonKADS methodology, MIT Press, Cambridge, Massachusetts, London, 2000.

[8] E. Zemmouri, H. Behja, and A. Marzak, "OntoECD : une ontologie pour le processus d'ECD," In proceedings of the WOTIC Workshop, Agadir, Morocco, december 24-25, 2009.

[9] A. Bernstein, F. Provost, and S. Hill, "Towards intelligent assistance for a data mining process: an ontology based approach for cost-sensitive classification," IEEE Transactions on Knowledge and Data Engineering 17(4), pp. 503-518, 2005.

[10] C. Diamantini, D. Potena, and E. Storti, "Ontology-driven KDD process composition,” N. Adams et al. (Eds.), LNCS, volume 5772, pp. 285-296, Springer 2009.

[11] T. Euler, "Publishing Operational Models of Data Mining Case Studies," Workshop on Data Mining Case Studies at the $5^{\text {th }}$ IEEE ICDM, pp. 99106, Houston, Texas, USA, 2005.

[12] K. Morik, and M. Scholz, "The MiningMart approach to knowledge discovery in databases," Intelligent Technologies for Information Analysis, Springer, pp. 47-65, 2004.

[13] M. Cannatro, and C. Comito, "A data mining ontology for grid programming," In proceedings of 1st International Workshop on Semantics in Peer-to-Peer and Grid Computing, in conjunction with WWW2003, pp. 113-134, 2003.

[14] M. Hilario, P., Do, H. Nguyen, A. Woznica, and A. Kalousis, "Ontology-based meta-mining of knowledge discovery workflows," In N. Jankowski, W. Duchs, and K. Grabczewski (Eds.), Studies in Computational Intelligence, Vol. 358/2011, Meta-Learning in Computational Intelligence, pp. 273-315, Springer Verlag, 2011.

[15] J-U. Kietz, F. Serban, and A. Bernstein, "eProPlan: A tool to model automatic generation of data mining workflows,". In P. Brazdil, A. Bernstein, and J-U. Kietz (Eds.), Proceedings of the 3rd Planning to Learn Workshop (WS9) at ECAI 2010, 2010.

[16] H. Behja, E. Zemmouri, A. Marzak, "Viewpoint-based Annotations for Knowledge Discovery in Databases", Proc. of IEEE ICMWI, p. 299302, Algiers, Algeria, 2010.

[17] A. I. Anton, "Goal based requirements analysis," In Proceedings of the $2^{\text {nd }}$ International Conference on Requirements Engineering ICRE'96, Colorado Springs, Colorado, pp. 136-144, 15-18 April 1996.

[18] A. I. Anton, Goal Identification and Refinement in the Specification of Information Systems, Ph.D. Thesis, Georgia Institute of Technology, June 1997.

[19] B. Nuseibeh and S. Easterbrook, "Requirements engineering: a roadmap," In Proceedings of the Conference on The Future of Software Engineering ICSE '00, ACM, New York, NY, USA, pp. 35-46, 2000.

[20] P. Giorgini, S. Rizzi, and M. Garzetti, "Goal-oriented requirement analysis for data warehouse design," In Proceedings of the 8th ACM international workshop on Data warehousing and OLAP (DOLAP '05), pp 47-56, ACM, New York, NY, USA, 2005.

[21] S. Khouri, L. Bellatreche, and P. Marcel, "Towards a Method for Persisting Requirements and Conceptual Models in Data Warehousing Context," 27ième Journées Bases de Données Avancées BDA2011, Rabat, Morocco, October 2011.

[22] M. Kumar, A. Gosain and Y. Singh, "Stakeholders Driven Requirements Engineering Approach for Data Warehouse Development," Journal of Information Processing Systems, Vol. 6(3), pp. 385-402, 2010.

[23] M. Glinz, "On Non-Functional Requirements," $15^{\text {th }}$ IEEE International Requirements Engineering Conference RE 2007, pp. 21-26, October 2007.

[24] R. Jarvis, G. McArthur, J. Mylopoulos, P. Rodriguez-Gianolli, and S. Zhou, "Semantic Models for Knowledge Management," In Proceedings of the Second International Conference on Web Information Systems Engineering WISE'01, Vol. 1, pp. 8-16, IEEE Computer Society, Washington, DC, USA, 2001.

[25] P. Giorgini, E. Nicchiarelli, J. Mylopoulous, and R. Sebastiani, "Formal Reasoning Techniques for Goal Models," Journal on Data Semantics, LNCS, Vol. 2800/2003, pp. 1-20, Springer, 2004.

[26] A. Aybuke and W. Claes, Engineering and Managing Software Requirements, Springer-Verlag Berlin, 2005.

[27] P. Chapman, J. Clinton, R. Kerber, T. Khabaza, T. Reinartz, C. Shearer, R. Wirth, "CRISP-DM 1.0 Stepby-step data mining guide," Technical report, CRISM-DM consortium, 1999.

[28] C. Rolland, and C. Salinesi, "Modeling Goals and Reasoning with Them," In A. Aurum, C. Wohlin (Eds.), Engineering and Managing Software Requirements (EMSR), Springer Verlag, 2005.

[29] $\mathrm{FaCT}++$, "FaCT++ OWL-DL reasoner," http://owl.man.ac.uk/factplusplus/ 